\title{
КЛИНИЧЕСКИЕ ХАРАКТЕРИСТИКИ ПАЦИЕНТОВ С ПЕРВИЧНЫМ ГИПЕРПАРАТИРЕОЗОМ: АНАЛИЗ ДАННЫХ ЭНДОКРИНОЛОГИЧЕСКОГО ОТДЕЛЕНИЯ
}

\author{
${ }^{1}$ Сапожникова И.Е., ${ }^{2}$ Веденская Т.П. \\ 'ФГБОУ ВО Кировский государственный медицинский университет, Киров \\ ${ }^{2}$ КОГБУЗ «Кировская областная клиническая больница»
}

ЦЕЛЬ: изучить клинические характеристики пациентов с первичным гиперпаратиреозом (ПГПТ) по данным эндокринологического отделения.

Объект исследования и методы. Проведено открытое ретроспективное исследование - анализ историй болезни лиц, госпитализированных в эндокринологическое отделение с 01.01.2013 г. по 31.12.2019 г. и имевших верифицированный ПГПТ (на основании гиперкальциемии в $\geq 2$ измерениях, повышенного уровня паратиреоидного гормона (ПТГ), визуализации околощитовидных желез (по данным УзИ, сцинтиграфии околощитовидных желез (ОЩЖ) с технецием пертехнетатом 99мТс (сестамиби) и/или компьютерной томографии), исключения третичного гиперпаратиреоза).

РЕЗУЛЬТАТЫ: критериям включения соответствовали 40 пациентов от 24 до 78 лет $(60,5$ [53,5;65,5] гг.): 36 (90\%) женщин и 4 (10\%) мужчин. Индекс массы тела (ИМТ) составил 28 [25;32] кг/м², концентрация общего холестерина - 5,06 [4,6;5,76] ммоль/л, триглицеридов - 1,16 [0,88;1,61] ммоль/л, холестерина липопротеидов низкой плотности - 3,13 [2,71;3.73] ммоль/л, холестерина липопротеидов высокой плотности - 1,38 [1,15;1,59] ммоль/л. Уровень общего кальция крови составил 2,85 [2,73; 3,08] ммоль/л, ионизированного кальция крови - $1,55\{1,45 ; 1,66\}$, неорганического фосфора крови - 0,76 [0,69; 0,86] ммоль/л, интактного ПТГ - 175,5 [118; 282] мг/мл (референсный диапазон 14-65 мг/мл). Манифестная форма ПГПТ диагностирована у 30 (75\%) пациентов, мягкая форма - у 10 (25\%) пациентов. Среди пациентов с манифестным ПГПТ костная форма обнаружена у 13 (32,5\%) пациентов, висцеральная форма у 8 (20\%) пациентов, смешанная (костно-висцеральная) форма ПГПТ - у 9 (22,5\%) обследованных лиц. Мочекаменная болезнь обнаружена у 16 (40\%) пациентов (из них у 2 (5\%) - в сочетании с язвенной болезнью двенадцатиперстной кишки). Признаки поражения костной ткани выявлены у $22(48,1 \%)$ пациентов, в том числе перенесенные малотравматичные переломы - у 7 (17,5\%) пациенток, гиперпаратиреоидная остеодистрофия - у 5 (12,5\%) пациенток (в одном случае - в сочетании с патологическим переломом). Длительность периода от манифестации типичных клинических проявлений до установления диагноза ПГПТ составила 6\{2;24\} месяцев. Стойкая артериальная гипертензия (АГ) имелась у 25 (62,5\%) пациентов: у 19 (76\% лиц с АГ) - 1-й степени, у 6 (24\% лиц с АГ) - 2-й степени. Амбулаторно постоянную антигипертензивную терапию получал 21 пациент (84\% лиц с АГ). Нарушения гликемии диагностированы у 11 (27,5\%) пациентов: сахарный диабет 2-го типа (СД-2) - у 4 (10\% группы), пограничные нарушения гликемии - у 7 (17,5\%), в том числе нарушенная толерантность к глюкозе в 5 случаях, нарушенная гликемия натощак - в 2 случаях. Стойкая АГ имелась у 8 (72,8\%) пациентов с нарушениями гликемии (в том числе у 4 лиц с СД-2), у 17 (58,6\%) пациентов без нарушений гликемии ( $p=0,49$, двусторонний вариант точного критерия Фишера). В зависимости от наличий нарушений гликемии пациенты с ПГПТ не различались по величине ИМТ ( $p=0,38)$, параметров липидного спектра $(p \geq 0,35)$ и степени $А Г(p=0,62$, двусторонний вариант точного критерия Фишера).

\section{выводы.}

1. Пациентов с ПГПТ характеризовали преобладание женщин (90\%), преимущественно средний возраст $(60,5[53,5 ; 65,5]$ лет).

2. Мягкая форма ПГПТ обнаружена у 10 (25\%) пациентов, манифестная - у 30 (75\%) пациентов, в том числе костно-висцеральная - у 9 (22,5\%), костная - у 13 (32,5\%), висцеральная - у 8 (20\%) пациентов.

3. Стойкая артериальная гипертензия выявлена у 25 (62,5\%) пациентов, нарушения гликемии у 11 (27,5\%) пациентов (в том числе у 4 (10\%) - СД-2). В зависимости от наличия нарушений гликемии пациенты не различались по частоте и степени АГ, ИМТ, уровням параметров липидного спектра. 